\title{
An Automated Evaluation of Regional Left Ventricular Function on Cine Magnetic Resonance Images
}

\author{
R EL Berbari ${ }^{1,2,3}$, N Kachenoura ${ }^{1,2}$, A Redheuil ${ }^{1,4}$, A Herment ${ }^{1,2}$, \\ I Bloch $^{3}$, E Mousseaux $^{1,4}$, F Frouin $^{1,2}$ \\ ${ }^{1}$ INSERM, UMR S 678, LIF, Paris, France \\ ${ }^{2}$ UPMC Univ Paris 06, UMR S 678, LIF, Paris, France \\ ${ }^{3}$ TELECOM Paris-Tech (ENST), TSI Department, CNRS UMR 5141, Paris, France \\ ${ }^{4}$ APHP, HEGP, Department of Cardiovascular Radiology, Paris, France
}

\begin{abstract}
Routine evaluation of the regional ventricular function in cardiac magnetic resonance (CMR) remains mostly based on visual analysis of cine loops and subsequent interobserver variability remains a potential drawback for diagnosis and follow-up of coronary artery disease (CAD). In this paper we propose a method that ensures automated assessment of normalized mean contraction times and radial endocardial velocities from MR cine images, by combining a parametric imaging analysis to an automatic segmentation method of the left ventricle. Short-axis cine loops were obtained in 20 subjects (10 myocardial infarction (MI) and 10 control). Reference segmental wall motion was scored by an expert according to 4 classes (normokinesia, hypokinesia, akinesia and dyskinesia). Results showed increased values for normalized mean contraction times and decreased values for the radial velocity in segments with wall motion abnormalities (WMA) compared to segments with normal function. These functional parameters could help to detect regional WMA.
\end{abstract}

\section{Introduction}

An accurate evaluation of the segmental contractile function remains central to the detection of coronary artery disease and to the evaluation of myocardial viability. Techniques evaluating segmental contraction should be minimally invasive, accurate and reproducible. Cine CMR allows to study wall thickening for the detection of regional wall motion abnormalities (RWMA) after myocardial infarction. In clinical context, RWMA is visually assessed. This necessitates a complex mental integration of parameters such as wall thickness and thickening, endocardial motion, myocardial deformation and temporal parameters leading to a decision making process influenced by experience level and which depends on individual appreciation. Quantitative methods have been proposed to decrease interpretation bias based on analysis of myocardial deformation on MR images [1,2].

The parametric analysis of main motion, first proposed in echocardiography [3] was adapted to cine short-axis MR images [4] and the estimation of regional parameters: mean contraction times and radial endocardial velocities was then introduced. This study showed significant differences in values of the estimated functional parameters between normal and pathological segments. However, to obtain these parameters, a delineation of the LV endocardial border at the end-diastolic phase of the cardiac cycle was necessary and was done manually by an expert in [4].

Therefore the goal of this work was to automate this segmentation step. Semi-automatic and automatic methods using various techniques of image processing [5] were proposed to detect the myocardial borders. However including the papillary muscles within the endocardial delineation remains a difficulty especially for gradient based methods because of the low gray value in the muscles, compared to the high value in the blood cavity. Prior knowledge learned from image models [6] can overcome these limits, but including papillary muscles in the endocardial surface really depends on the model which is in general created by manual segmentation. Besides, these techniques necessitate heavy numerical computation and are not simple to be used.

In a previous paper [7], we applied the active contour approach using the GVF snake on filtered images by connected morphological operators, in order to include the papillary muscles into the cavity. The proposed method showed to be robust and simple to be used in a clinical setting since the same parameters were used for each sub- 
ject. This automatic segmentation method was then integrated in the quantification process in order to estimate automatically functional parameters on normal subjects [8]. The validation was done by showing that similar values of the functional parameters were obtained using either manual or automatic segmentation. Furthermore, this study demonstrated that the mean contraction time parameter $T_{m c}$ was correlated with the heart cycle duration. Thus the normalized mean contraction time $F_{m c}$ was defined as the ratio of $T_{m c}$ to the R-R interval duration. This parameter provided more homogenous values and made the comparison between subjects easier.

The goal of the actual study was to automatically estimate normalized mean contraction times and radial endocardial velocities on patients with myocardial infarction and study the differences with control subjects. In Section 2 the main steps of the quantification process and the segmentation method are described. Results of the estimated parameters on both control and MI groups are presented in Section 3 and finally discussed in Section 4.

\section{Materials and methods}

\subsection{Population and acquisition protocol}

Ten healthy subjects ( 9 men/ 1 women, mean age $=48.8$, range $=40-60$ ) having no prior cardiovascular history and ten patients admitted for myocardial infarction $(7 \mathrm{men} / 3$ women, mean age $=57.2$, range $=39-78$ ) were included in the study. Contraction cine loops with ECG gating were obtained using Fast Imaging Employing Steady State Acquisition (FIESTA), a steady state free precession (SSFP) technique, according to a standard protocol on a $1.5-\mathrm{T}$ MRI system (Signa LX, GE Medical Systems, Milwaukee, WI) with an 8-element thoracic phased-array surface coil. All MR images were acquired using breath-holding. Cine loops were acquired in eight to fourteen short-axis views from the mitral annulus to the apex using the following parameters: TR: $3.7-4 \mathrm{~ms}$, TE: $1.6-1.7 \mathrm{~ms}$, flip angle: $50^{\circ}$, cardiac phases: $20-40$ after view sharing, slice thickness: $8 \mathrm{~mm}$, inter slice gap: $1 \mathrm{~mm}$, imaging matrix: $512 \times 512$ pixels, FOV: $30-38 \mathrm{~cm}$. The pixel size was between $0.75 \times 0.75$ and $1.7 \times 1.7 \mathrm{~mm}^{2}$ and the delay between two successive frames between 20 and $40 \mathrm{~ms}$.

\subsection{Reference segmental wall motion as- sessment}

Two points, the left ventricle center $P_{0}$ and the anterior intersection between the right and left ventricles $P_{1}$, were defined on the end-diastolic image by an operator. The distance $\operatorname{dist}\left(P_{0}, P_{1}\right)$ served to define a region of interest (ROI). Using these two points, six angular sectors were automatically traced according to the recommended model
[9] as: anterior (A), anterolateral (AL), inferolateral (IL), inferior (I), inferoseptal (IS), and anteroseptal (AS) segments. For all the studies, the segmental wall motion score was assessed by an expert, according to a four-point scale (normokinesia, hypokinesia, akinesia, and dyskinesia).

\subsection{Automated endocardial delineation}

Endocardial delineation encounters a primordial difficulty due to problems with papillary muscles and the poor homogeneity of the cavity. The segmentation method [7] consists of a filtering step applied to the original image using alternately area-opening and area-closing filters, followed by the GVF snake algorithm. An optimal choice of the filter parameter $\lambda$ is done as described in [7]. Applied to CMR images, the filtering step allows to merge the papillary muscles corresponding to sets of low gray level with the blood cavity zone corresponding to high gray levels. The deformation process of the GVF snake is controlled by five parameters: $\alpha$ that controls the elasticity of the contour, $\beta$ its rigidity, $\kappa$ weights the external force that includes a pressure force weighted by $\kappa_{p}$ and a Gradient Vector Flow force regulated by the parameter $\mu_{g v f}[10]$.

A first contour was defined on the filtered images using $P_{0}$ as an initialization and the GVF snake method with the following parameters: $(\alpha=1, \beta=60, \kappa=1.6$, $\kappa_{p}=0.5$ and $\left.\mu_{g v f}=0.3\right)$, the high value of $\beta$ generates a rigid contour englobing the papillary muscles into the cavity. A refinement of this segmentation was necessary in order to recover boundary information and catch all details that might be lost during the filtering process. Thus, the first contour was used as initialization of a new segmentation, applied to the gradient of the original image with lower values of $\beta=5, \kappa_{p}=0.1$ and $\mu_{g v f}=0.1$. This segmentation method was validated on control subjects and patients $[7,11]$.

\subsection{Parametric analysis of main motion}

For each pixel, the time-signal intensity curve $P(x, y, t)$ was observed to study the intensity variation during the cardiac cycle. The intensity of a pixel into the cavity during the diastolic phase, $A_{B}(x, y)$ decreases to an intensity level corresponding to the myocardium $\left(A_{B}(x, y)-\right.$ $\left.A_{V}(x, y)\right)$ during the systolic phase. This curve is fitted to a nonlinear transition model defined by an adaptive window function $g(t)$ as follows:

$$
\begin{gathered}
P(x, y, t)=A_{B}(x, y)- \\
A_{V}(x, y) \cdot g\left(t, T_{O N}(x, y), T_{O F F}(x, y)\right)+e(x, y, t)
\end{gathered}
$$

where $A_{B}(x, y)$ is the signal intensity at the end-diastole, $A_{V}(x, y)$ the variation of signal intensity, $T_{O N}(x, y)$ the starting time of the contraction, $T_{O F F}(x, y)$ the ending 
time of the contraction and $e(x, y, t)$ is the residual error. From this model the parametric images related to motion amplitude $A_{B}$ and $A_{V}$ and contraction times $T_{O N}$ and $T_{O F F}$ were automatically computed [4]. A mean contraction time image was calculated as the average between the $T_{O N}$ image and the $T_{O F F}$ image. The end-diastolic contour obtained with the method described above (Section 2.3) was used to limit the six segments described in Section 2.2. Segmental mean contraction times, $T_{m c}$ and their normalized values $F_{m c}$ were thus computed inside these regions. Moreover, using the endocardial end-diastolic contour, a distance map was computed and combined with the $T_{O N}$ image in order to estimate the radial component of the endocardial velocity, $V_{m}$ as the slope of a linear regression of the plot showing for each pixel, its distance to the contour versus its $T_{O N}$ value.

\section{Results}

Figure 1 shows a result of the segmentation on one subject with 8 slices from the apex to the base. This figure shows the robustness of the applied method against the heterogeneity of the cavity on apical levels and the presence into the cavity of papillary muscles on mid ventricle slices.

In the control group, the $F_{m c}$ parameter was estimated

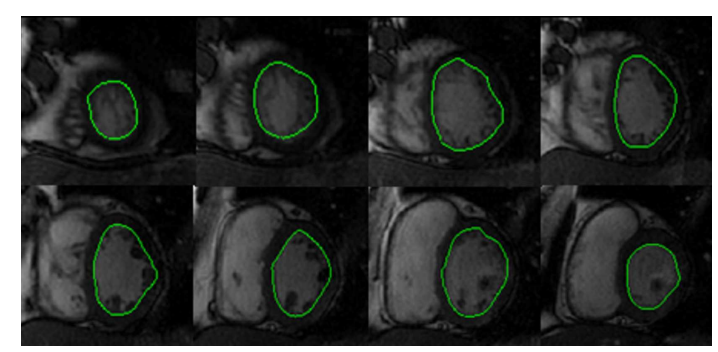

Figure 1. Result of the automatic segmentation, for 8 slices from the apex (top left) to the base (bottom right) in one subject.

in $99.8 \%$ of the segments and the radial velocities $V_{m}$ in $69.4 \%$. Overall, a mean values and a standard deviations of $0.40 \pm 0.06$ were found for the normalized mean contraction times and $5.36 \pm 2.16 \mathrm{~cm} / \mathrm{s}$ for the radial velocities.

For patients with MI, the $F_{m c}$ parameter was estimated in $97.3 \%$ of the segments and the radial velocities in $67.6 \%$. In this group, segments were classified according to 3 classes: MI-Normal $(n=169)$, with mean values \pm standard deviations of $V_{m}$ equal to $5.53 \pm 2.12 \mathrm{~cm} / \mathrm{s}$ and mean values of normalized mean contraction times equal to $0.41 \pm 0.06$, MI-Hypo $(\mathrm{n}=122)$ with values of $4.22 \pm 1.87 \mathrm{~cm} / \mathrm{s}$ and $0.45 \pm 0.07$ and finally the class MIAdys $(n=117)$ that contains akinetic and dyskinetic segments on the MI group with values of $4.07 \pm 2.16 \mathrm{~cm} / \mathrm{s}$ and
$0.51 \pm 0.09$. Similar values were obtained in MI-Normal and control group.

Statistical analysis using Student's t-test showed a significant difference between MI-normal and MI-Hypo classes on the normalized mean contraction times $(p<$ $\left.10^{-6}\right)$ and the radial endocardial velocities $\left(p=10^{-6}\right)$ and between MI-normal and MI-Adys classes on the $F_{m c}$ values $\left(p<10^{-6}\right)$ and on the $V_{m}$ values $\left(p=10^{-4}\right)$, (Figure 2). Moreover, a significant difference on the $F_{m c}$ values was observed between the two last classes MI-Hypo and MI-Adys $\left(p=10^{-6}\right)$.
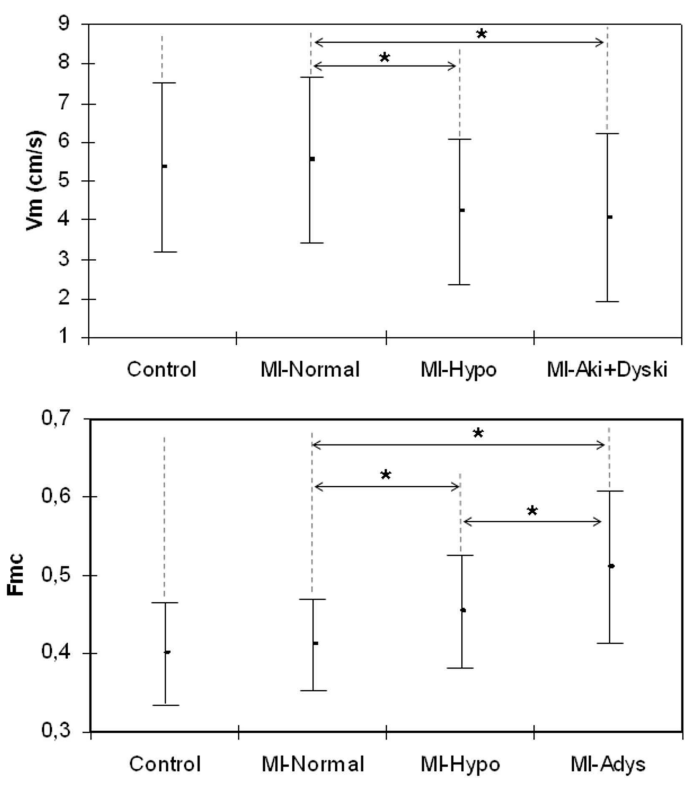

Figure 2. Mean values and standard deviations of quantitative parameters: $V_{m}$ and $F_{m c}$ extracted from control group, normal segments of the MI group (MI-Normal), hypokinetic segments of the MI group (MI-Hypo), akinetic and dyskinetic segments of the MI group (MI-Adys) classes; $(*)$ indicates a statistically significant difference between two classes.

\section{Discussion and conclusions}

The visual evaluation of the regional left ventricular function is based on endocardial excursion and myocardial thickening. Since the visual assessment of wall motion is experience dependent and subjective, quantitative techniques have been developed using manual tracing of the endocardial borders.

In this paper, a robust segmentation method, which includes papillary muscles and takes into account the heterogeneity of the gray levels due to blood flow in the LV cavity, was applied to a set of data composed of ten control subjects and ten patients with myocardial infarction. This 
method is simple to use since it requires only the definition of two points on the end-diastolic image and a simple setting of the snake parameters. Using the resulting enddiastolic contour, the quantification method presented in [4] is largely automated. Thus normalized mean contraction times and radial endocardial velocities were estimated automatically.

Differences between normal and infarcted segments that were proved in [12] and [4] are confirmed here where both increased values for the normalized mean contraction times and decreased velocities in segments after myocardial infarct were observed when they are compared to values obtained on normal segments. When classifying the segments according to the different classes given by the expert, we can observe a link between the parameter values on a segment and the class to which it belongs. The more the contractile abnormality degree increases in the segment, the more the normalized mean contraction time increases. We should notice here that it was more difficult to estimate the velocity (in $67 \%$ and $69 \%$ of the segments) than the normalized time parameter that showed to be more robust (estimated in $97 \%$ and $99 \%$ of the segments). Moreover, the coefficients of variation on this time parameter are smaller than the coefficients variation on the velocity. Furthermore, the normalized mean contraction time $F_{m c}$ is more sensitive for the description of wall motion abnormalities, since it highlights a significant difference ( $p=10^{-6}$ ) between the two classes MI-Hypo and MIAdys (Figure 2) that was not obtained on the mean contraction time $T_{m c}$ in [4].

All these results encourage us to estimate these parameters on larger series of patients. They allow us to take advantage of routine cine sequences without the need for specific tagged acquisitions thereby significantly reducing examination time.

In conclusion, the normalized mean contraction time and radial endocardial velocity parameters can be derived from SSFP cine images by a robust procedure which requires a very reduced number of interactions with a nonexpert user. These functional parameters have a potential to quantify regional myocardial contraction and could help to identify motion abnormalities in clinical studies.

\section{References}

[1] Caiani E, Toledo E, MacEneaney P, Collins K, Lang R, Mor-Avi V. The role of still-frame parametric imaging in magnetic resonance assessment of left ventricular wall motion by non-cardiologists. J Cardiovasc Magn Reson 2004; 6:619-625.

[2] Redheuil A, Kachenoura N, Laporte R, Azarine A, Lyon $\mathrm{X}$, Jolivet O, Frouin F, Mousseaux E. Interobserver variability in assessing segmental function can be reduced by combining visual analysis of $\mathrm{cmr}$ cine sequences with cor- responding parametric images of myocardial contraction. J Cardiovasc Magn Reson 2007;9:863-872.

[3] Ruiz-Dominguez C, Kachenoura N, De Cesare A, Delouche A, Lim P, Gerard O, Herment A, Diebold B, Frouin F. Assessment of left ventricular contraction by parametric analysis of main motion (pamm): theory and application for echocardiography. Phys Med Biol 2005;50:3277-3296.

[4] Kachenoura N, Redheuil A, Balvay D, Ruiz-Dominguez C, Herment A, Mousseaux E, Frouin F. Evaluation of regional myocardial function using automated wall motion analysis of cine mr images: Contribution of parametric images, contraction times, and radial velocities. J Magn Reson Imaging 2007;26:1127-1132.

[5] Frangi A, Niessen W, Viergever M. Three-dimensional modeling for functional analysis of cardiac images: A review. IEEE Transactions on Medical Imaging 2001;20:225 .

[6] Kaus M, von Berg J, Weese R, Niessen W, Pekar V. Automated segmentation of the left ventricle in cardiac mri. Medical Image Analysis 2004;8:245-254.

[7] El Berbari R, Frouin F, Redheuil A, Angelini E, Mousseaux E, Bloch I, Herment A. Développement et évaluation d'une méthode de segmentation automatique de l'endocarde sur des images acquises par résonance magnétique. ITBM RBM 2007;28:117-123.

[8] El Berbari R, Kachenoura N, Redheuil A, Giron A, Mousseaux E, Herment A, Bloch I, Frouin F. An automated estimation of regional mean contraction times and radial velocities from cine magnetic resonance images. evaluation in normal subjects. Journal of Cardiovascular Magnetic Resonance 2008;Submitted.

[9] Cerqueira M, Weissman N, Dilsizian V, Jacobs A, Kaul S, Laskey W, Pennell D, Rumberger J, Ryan T, Verani M. Standardized myocardial segmentation and nomenclature for tomographic imaging of the heart: a statement for healthcare professionals from the cardiac imaging committee of the council on clinical cardiology of the american heart association. Circulation 2002;105:539-542.

[10] Xu C, Prince J. Snakes, shapes, and gradient vector flow. IEEE Trans Med Imag 1998;7:359-369.

[11] El Berbari R, Bloch I, Redheuil A, Angelini E, Mousseaux E, Frouin F, Herment A. Automated segmentation of the left ventricle including papillary muscles in Cardiac Magnetic Resonance images. In FIMH, volume 4466. 2007; 453462.

[12] Lucats L, Monnet X, Bize A, Chetboul V, Pouchelon J, Hittinger L, Berdeaux A, Ghaleh B. Regional and temporal heterogeneity of postsystolic wall thickening is associated with left ventricular asynchrony in normal and experimental stunned myocardium. Basic Res Cardiol 2008;103:385396.

Address for correspondence:

Racha EL Berbari

INSERM U678 LIF, 91 bd de l'Hopital, 75013 Paris, France

Racha.ElBerbari@imed.jussieu.fr 